\title{
Autophagy controls programmed death-ligand 1 expression on cancer cells (Review)
}

\author{
LIJUAN GAO $^{1,2}$ and YONGSHUN CHEN ${ }^{1,2}$ \\ ${ }^{1}$ Department of Clinical Oncology, Renmin Hospital of Wuhan University; \\ ${ }^{2}$ The First Clinical College of Wuhan University, Wuhan, Hubei 430060, P.R. China
}

Received January 13, 2021; Accepted April 29, 2021

DOI: $10.3892 /$ br.2021.1460

\begin{abstract}
Programmed death-ligand 1 (PD-L1) is a transmembrane protein mainly located on cancer cells, including renal cell carcinoma, breast, colorectal, gastric and non-small cell lung cancer. PD-L1 binds to the PD-1 receptor expressed on T lymphocytes to inhibit the activation of $\mathrm{T}$ lymphocytes, thus allowing tumour cells to escape immune surveillance, leading to tumour growth and the poor prognosis of patients with cancer. Inhibitors targeting the programmed death-1/PD-L1 axis have been widely used in the clinical treatment of a variety of solid tumours in recent years. However, the clinical efficacy of these inhibitors varies. Studies have demonstrated that the effect of the targeted drug is positively associated with the expression of PD-L1 on the tumour membrane. Hence, exploring the mechanism of PD-L1 expression is very important for the treatment of tumours. Autophagy is a physiological process that maintains the stability of the internal environment. Autophagy degrades aging organelles and long-lived proteins and produces nutrients for cell recycling. To the best of our knowledge, the present review is the first to summarize the research that has been conducted on autophagy-regulated PD-L1 expression, which may provide new avenues for tumour immunotherapy.
\end{abstract}

\section{Contents}

1. Introduction

2. PD-L1 expression level is a potential clinical biomarker for PD-1/PD-L1 antibodies

3. Mechanisms of autophagy that regulate the expression of PD-L1

4. Discussion

Correspondence to: Professor Yongshun Chen, Department of Clinical Oncology, Renmin Hospital of Wuhan University, 238 Jiefang Road, Wuhan, Hubei 430060, P.R. China

E-mail: yongshun2007@163.com

Key words: programmed death-ligand 1, autophagy, tumour immunotherapy

\section{Introduction}

Programmed death-ligand 1 (PD-L1), with the gene name CD274, was first discovered in interleukin (IL)-3-deprived LyD9 (murine hematopoietic progenitor) and 2B4-11 (murine $\mathrm{T}$ cell hybridoma) cell lines in 1992 (1) and was described as B7-H1 by Dong et al (2) in 1999. PD-L1 is the third member of the B7 family that does not bind CD28, cytotoxic T-lymphocyte A4 or inducible co-stimulator, and has 10-25\% homology with B7.1 and B7.2 proteins (2). PD-L1 is encoded by the PDCDL1 gene, which was discovered at 24.1 on human chromosome 9. The amino acid sequence of PD-L1 is encoded by 7 exons, which form a protein of $\sim 40 \mathrm{kDa}$. PD-L1 is a type I transmembrane protein, is part of the immunoglobulin (Ig) superfamily and is composed of IgV-like and IgC-like extracellular domains, a hydrophobic transmembrane domain and a short cytoplasmic tail composed of 30 amino acids. The signal transduction mechanisms of PD-L1 remain unclear (3). The most important role of PD-L1 is binding with programmed death-1 (PD-1; CD279), a type I transmembrane receptor that is 288 amino acids long and was first found on T cells (4). The engagement of PD-L1 and PD-1 on cancer cells activates Src homology region 2 domain-containing phosphatases, which inhibit the T cell receptor (TCR) pathway. Inhibition of the TCR pathway leads to inhibition of T cell activities, including proliferation, survival and cytokine production, such as that of IL-2, tumour necrosis factor $\alpha$ (TNF- $\alpha$ ) and interferon $\gamma$ $(\mathrm{IFN}-\gamma)(5)$, as well as the inhibition of B7-1 and T cell tolerance $(6,7)$. To the best of our knowledge, the present review will discuss for the first time how autophagy, a protein degradation pathway that regulates homeostasis of cells, also regulates PD-L1 expression on cancer cells.

\section{PD-L1 expression level is a potential clinical biomarker for PD-1/PD-L1 antibodies}

Considering the role of PD-L1 in suppressing the activation of $\mathrm{T}$ cells, it has been an outstanding target for targeted tumour therapy during the past few years (8). To be specific, obstructing the PD-L1/PD-1 signalling pathway by antibodies can reactivate exhausted $\mathrm{T}$ cells in the tumour microenvironment, thus making tumour cells vulnerable to attack by cytotoxic T cells (6). Currently, the efficacy and safety of drugs targeting the PD-L1/PD-1 axis have been identified in 
$>1,000$ clinical trials, and these drugs have been authorised for the treatment of various cancer types, including melanoma (9), Hodgkin's lymphoma (10), non-small cell lung cancer (NSCLC) (11), microsatellite instability-high renal cell carcinoma (12), deficient mismatch repair cancer (13), urothelial carcinoma (14), Merkel cell carcinoma (15), hepatocellular carcinoma (16), gastric cancer (17) and head and neck squamous cell carcinoma (18). Even so, in some tumour types, such as non-MSI (microsatellite instability) colorectal cancer, prostate cancer, ovarian cancer and breast cancer, the clinical efficacy of the drugs targeting PD-1/PD-L1 when used alone was limited (19). The factors that influenced the effective response of PD-1/PD-L1 include tumour-infiltrating lymphocyte (TIL) infiltration and localisation at an early stage (20), TIL activation level (21) and the effect of mutations in tumour cells (22). The expression level of PD-L1 is the first factor notably associated with prognosis and clinical effects of the drugs targeted to PD-1/PD-L1 among various tumours such as melanoma, gastric cancer and NSCLC (23-25). Among the 45 FDA-approved drugs across 15 tumour types, PD-L1 was predictive in only $28.9 \%$ of cases, and was either not predictive $(53.3 \%)$ or not tested (17.8\%) in the remaining cases. This indicates that PD-L1 has some limitations as a predictive biomarker; however, it still serves a notable role as a biomarker in bladder, NSCLC, triple-negative breast and cervical cancer (26). The expression of PD-L1 was found to be increased among different solid tumours and was associated with worse survival, prognosis and treatment responses in multiple malignancies such as esophageal, gastric, urothelial and colorectal cancer and hepatocellular carcinoma $(27,28)$, mostly due to the upregulation of PD-L1, which created a favourable environment for tumour progression by inhibiting antitumour immunity $(29,30)$. The mechanisms that regulate the expression of PD-L1 remain unclear. Hence, it is imperative to broaden understanding of the regulation of PD-L1 expression in order to improve the therapeutic efficacy of current immune checkpoint blockade drugs, such as pembrolizumab and nivolumab. In addition, greater understanding will improve tumour immunotherapy, which will lead to an improved prognosis in patients with cancer.

\section{Mechanisms of autophagy that regulate the expression of PD-L1}

Autophagy is a tightly coordinated process that isolates misfolded or mutated proteins, damaged or aged organelles into a double membrane vesicle called an autophagosome. Autophagosomes fuse with lysosomes, forming autolysosomes, the contents of which can then be used to degrade the components of the autolysosomes (31). Nucleotides, amino acids and other nutrients produced by the aforementioned degradation process can be recycled by cells. The recycling capacity of autophagy is conserved from yeast to humans and regulates cellular homeostasis in both physiological and pathophysiological contexts. Currently, autophagy has been classified into 3 forms: i) Macroautophagy, ii) microautophagy and iii) chaperone-mediated autophagy (CMA). Among these, macroautophagy is the dominate type of autophagy, and is a common process for the degradation of cytoplasmic components and organelles for nutrient recovery in cell recycling.
However, microautophagy is a non-selective degradation process that directly swallows intracellular components into tubules or lysosomes (32). CMA is unlike the other two aforementioned types of autophagy in that it only participates in the degradation of soluble proteins that contain the KFERQ sequence motif, such as perilipin 2 and pirilipin3 (33). After being recognised by the $70-\mathrm{kDa}$ cytoplasmic heat shock protein (Hsc70), a complex is formed by these proteins in combination with Hsc70 and its chaperones, which are transported to the lysosome and interact with the lysosomal associated membrane protein-2A receptor and are degraded by acid hydrolase in lysosomes (34).

The process of autophagy is achieved in 4 distinct stages: i) Initiation, ii) nucleation, iii) maturation and iv) degradation (35). First is the process of autophagosome initiation, which is controlled by the Unc-51-like kinase 1 (ULK)-autophagy-related gene 13 (Atg13)-family interacting protein $200 \mathrm{kDa}$ kinase complex. This complex is negatively regulated by the mammalian target of rapamycin complex 1 (mTORC1) via activation of ULK and AMP-activated protein kinase (AMPK) (36). Second, the phagophore nucleation step is achieved by the phosphatidylinositol 3-kinase (PI3K) complex, Beclin 1, Atg14L, Vps (vesicular protein sorting) 15 , Vps 34 , UV radiation resistance associated gene and Bax interacting factor 1 (36). The proteins participating in the process of initiation and nucleation can synergistically facilitate the formation of the double membrane structure of autophagosomes (36). The type of membranes can originate from the mitochondria, plasma membrane or the endoplasmic reticulum $(37,38)$. Third, is the elongation or expansion step, in which the Atg5-Atg12-Atg16 complex is formed to elongate the autophagosome double membranes. Simultaneously, members of the g-aminobutyric acid receptor-associated protein and LC3 families of proteins are recruited to the membrane after binding to the lipid phosphatidylethanolamine (PE). LC3 (Atg8)-I is cleaved by Atg4 and in turn conjugated with PE by Atg3 and Atg7 to form LC3-II (also known as MAP1LC3B). LC3-II can serve as a marker to exhibit the quantity of autophagosomes formed at all points of the process. Finally, autophagosomes fuse with the lysosomes to degrade the substances in autophagosomes by the action of acid proteases in lysosomes. Moreover, this promotes energy efficiency through ATP generation and attenuates damage to the cell by removing non-functional proteins and organelles (35).

In 2019, Huang et al (39) found that autophagy can decrease PD-L1 expression both in vivo and in vitro. First, in caecal ligation and perforation (CLP) mice, with time after CLP, the LC3 expression in the lung tissue exhibited a downward trend, while the expression of PD-L1 increased markedly. When autophagy was induced by rapamycin (RAP) pretreatment, which enhanced the expression of LC3, the expression of PD-L1 was decreased in CLP mice. Similarly, in an in vitro experiment, when autophagy was inhibited by lipopolysaccharide at different time points, the expression of PD-L1 was induced at both mRNA and protein levels in mouse neutrophils when compared with that in the blank control group, and peaked at $12 \mathrm{~h}$ to levels significantly higher compared with those of the blank control group. The expression of LC3 and p62 was also increased at the same time. However, when combined with the autophagy inducer RAP, the expression of 


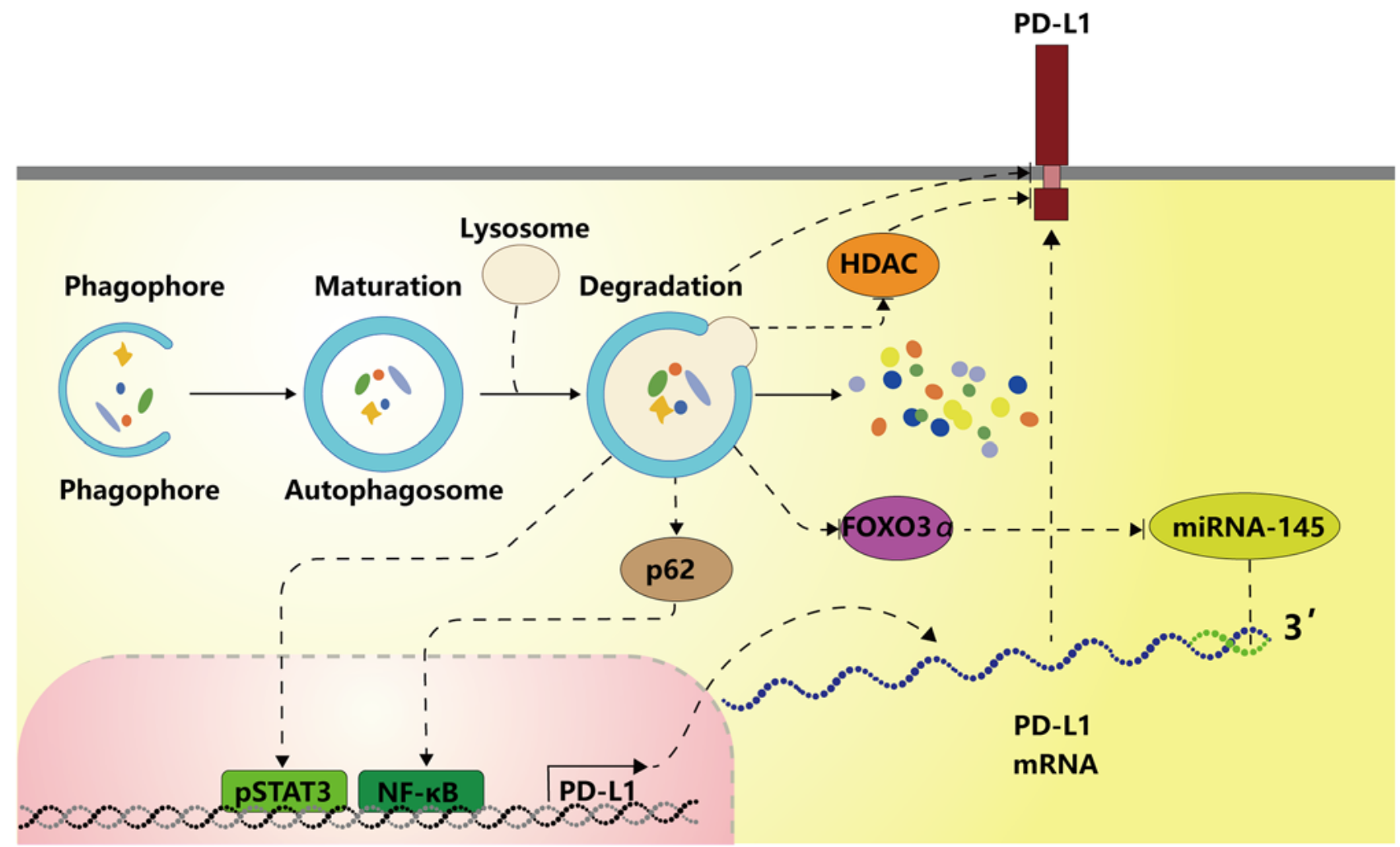

Figure 1. Mechanism of autophagy control. Autophagy controls PD-L1 expression via the histone deacetylases, p62/sequestosome-1/NF-kB pathway, STAT3 phosphorylation, ATG7/autophagy/FOXO3A/miR-145 axis and autophagy flux in cancer cells. PD-1, programmed death-1; miRNA/miR, microRNA; PD-L1, programmed death ligand-1; p, phosphorylated; HDAC, histone deacetylase.

PD-L1 and p62 was significantly downregulated, while the expression of LC3 was continually increased in mouse neutrophils when compared with the blank control group (39). The aforementioned results indicated that the process of autophagy may regulate PD-L1 expression via various pathways. In recent years, numerous studies have contributed to elucidating the mechanisms through which autophagic flux can influence PD-L1 expression, as described below. The mechanism is summarized in Fig. 1.

Autophagy decreases the expression of histone deacetylases (HDACs) to downregulate PD-L1 expression. Booth et al (40) found that exposure of lung and ovarian tumour cells (' $\mathrm{H}$ ' series NSCLC lines, SKOV3, OVCAR and PAI cells) to pemetrexed + sildenafil in vitro decreased the expression of various HDACs (HDAC2, HDAC4, HDAC6 and HDAC9), which could be blocked through the knockdown of crucial autophagy-related proteins (AMPKa, Beclin1 or ATG5). The degradation of histone deacetylases by pemetrexed + sildenafil is dependent on autophagy. Simultaneously, pemetrexed + sildenafil downregulated the expression of PD-L1, PD-L2 and ornithine decarboxylase (ODC), while upregulating the expression of MHC class I on lung and ovarian tumour cells. By contrast, the killing ability of pemetrexed + sildenafil was heightened by the histone deacetylase inhibitors AR42 and sodium valproate. At the same time, AR42 and valproate use alone quickly decreased the expression of PD-L1, PD-L2 and ODC, while increasing the expression of MHC class I and ceramide synthase 6 . Correspondingly, treatment of syngeneic mouse lung cancer cells in vitro with pemetrexed + sildenafil enhanced the clinical effects of immune checkpoint inhibitors targeting PD-1 or CTLA4 (cytotoxic T lymphocyte-associated protein 4). The aforementioned data demonstrated that exposing tumour cells to (pemetrexed + sildenafil) leads to tumour cell death and autophagy-dependent downregulation of HDACs and PD-L1 and opsonises the remaining tumour cells to target antitumour immunotherapy antibodies (40).

Dent et al (41) also found that the curcumin + sildenafil drug combination caused autophagosomes to form in colon cancer cells. Curcumin + sildenafil downregulated the expression of numerous HDAC proteins, such as HDAC2 and HDAC 3 , in an autophagy-dependent manner in colon cancer cells (41). In addition, molecular-knockdown of HDAC2, HDAC 3, or HDAC2 + HDAC3 by transfection of small interfering RNA notably inhibited the expression of PD-L1 and induced the expression of MHC class I (41), which demonstrated that the curcumin + sildenafil-induced decrease in PD-L1 expression is reliant on autophagy-mediated HDAC degradation.

Chen et al (42) found that a new HDAC6 inhibitor, MPT0G612, activated autophagy accompanied by LC3B-II upregulation and p62 downregulation to downregulate PD-L1 protein expression, stimulated by IFN- $\gamma$ in colorectal cancer (CRC) cells. In addition, the findings of the aforementioned study demonstrated that MPT0G612 significantly suppressed proliferation and viability, and induced apoptosis of CRC cells, hence exhibiting promising antitumour activity (42). Notably, inhibiting the autophagy process by small molecular inhibitors and shAtg5 transfection promoted MPT0G612-enhanced cell apoptosis (42). In addition, inhibiting HDAC6 expression decreased the MPT0G612-induced autophagy in CRC cells 
to further induce tumour cell apoptosis (42). These results demonstrated that MPT0G612 can induce cell death through inhibition of the HDAC6-associated pathway, and hence it may be a promising drug for enhancing immune checkpoint inhibition for the treatment of CRC.

Booth et al (43) found that the new antitumour drug GZ17-6.02 can kill gastrointestinal (GI) tumour cells and induce autophagy to downregulate PD-L1 expression. GZ17-6.02 augments autophagosome formation by activating ataxia-telangiectasia mutated (ATM), which is responsible for phosphorylating nuclear $\gamma \mathrm{H} 2 \mathrm{AX}$ and AMPK $\alpha$ T172. The activation of the ATM-AMPK signalling pathway contributed to the subsequent inhibition of mTOR and dephosphorylation of ULK1 S757 to induce autophagy. On interaction with 5-fluorouracil, GZ17-6.02 as an additive killed all the tested GI tumour cells and contributed to increased ATM phosphorylation, enhanced mTOR inactivation and autophagosome formation. Consequently, multiple HDAC proteins and chaperone proteins (HDAC2, HDAC4, HDAC6,HDAC9 and heat shock protein 70) were degraded via autophagy, and downregulation of HDAC led to decreased PD-L1, ornithine decarboxylase and indole amine 2,3-dioxygenase expression, and to the elevated expression of MHC class I. The curative effect of the anti-PD-1 checkpoint inhibitory antibody (anti-PD-1 endotoxin-free antibodies) was strengthened when GZ17-6.02 was used (43).

In another study, Booth et al (44) found that treatment of NSCLC cells with neratinib + HDAC inhibitors (sodium valproate) enhanced autophagosome formation and subsequently autolysosome formation by inhibiting the activation of mTORC1 and mTORC2, which caused an additive induction of cell apoptosis. In addition, knockdown of critical proteins Beclin1 or ATG5 inhibited HDAC inhibitors and neratinib from decreasing the expression of ERBB1/3/4 and K-/N-RAS, and impaired the neratinib + HDAC inhibitor curative effect. Neratinib + HDAC inhibitors decreased the expression of a variety of HDAC proteins through autophagy, which led to a decrease in the expression of PD-L1, PD-L2 and ornithine decarboxylase, and enhanced expression of MHC class I (44). As neratinib is approved for the treatment of breast cancer, it was selected to perform definitive animal studies in the highly aggressive 4T1 TNBC mammary carcinoma isolate. Notably, the study found that neratinib + HDAC inhibitors could inhibit the proliferation of $4 \mathrm{~T} 1$ breast tumours in vivo, which strengthened the effect of anti-PD-1 antibody.

Autophagy regulates PD-L1 expression via the p62/sequestosome (SQSTM)-1/NF- $\kappa B$ pathway. Wang et al (45) discovered that autophagy regulates PD-L1 expression via the p62/SQSTM1-NF- $\mathrm{B}$ signalling pathway in gastric cancer. First, autophagy inhibition by small molecular inhibitors and small interfering RNAs revealed that the PD-L1 expression levels were increased in vitro as well as in vivo. In addition, inhibition of autophagy enhanced PD-L1 expression promoted by IFN $-\gamma$. While IFN- $\gamma$ itself can simultaneously induce autophagy (46), it was found that IFN- $\gamma$ significantly upregulated PD-L1 expression via activation of the STAT1 signalling pathway independent of autophagy (46). Through western blotting and flow cytometry, it was demonstrated that inhibiting autophagy resulted in the accumulation of p62/SQSTM1 expression and increased NF- $\kappa$ B activation.
Correspondingly, inhibition of $\mathrm{NF}-\kappa \mathrm{B}$ and knockdown of p62/SQSTM1 restored overexpression due to blocking of autophagic flux. Finally, by using immunohistochemical staining, it was demonstrated that the protein levels of LC3 and p62/SQSTM1 were positively associated with PD-L1 expression among 137 primary tumour samples of gastric cancer. The expression level of PD-L1 was also positively associated with the number of infiltrating $\mathrm{T}$ cells (45).

Suppression of autophagy upregulates PD-L1 expression by promoting STAT3 phosphorylation. Tang et al (47) found that STAT3 was phosphorylated by microRNA (miR)-3127-5p through inhibition of autophagosome formation, leading to upregulation of PD-L1 expression in NSCLC cells. Knocking down miR-3127-5p induced the protein expression of LC3, while p62 protein expression decreased markedly, indicating an enhanced autophagic flux. By contrast, when miR-3127-5p was overexpressed in NSCLC cells, autophagy was found to be weakened compared with NSCLC cells in which miRNA-3127-5p had been knocked down (47). When autophagy was induced by RAP in the miR-3127-5p overexpressed cells, LC3 protein expression was enhanced, while the protein expression of p62 and pSTAT3 was decreased notably (47). When autophagy was inhibited by wortmannin in the miR-3127-knockdown cells, it was observed that LC3 protein expression was decreased, while the expression of p62 and pSTAT3 increased significantly (47). In addition, the miR-3127-5p-regulated elevated level of PD-L1 expression contributed to immune escape and the chemoresistance of lung cancer (47).

Autophagy increases PD-L1 mRNA stability and expression via the ATG/autophagy/FOXO3A/miR-145 axis. Zhu et al (48) found that the overexpression of ATG7 facilitated autophagic degradation of FOXO3 in bladder cancer and that the latter inhibited miR-145 transcription, hence elevating PD-L1 expression. The results revealed that lower expression of miR-145 decreases its direct engagement to the pd-11 mRNA 3'-UTR, thus increasing the stability and expression of pd-11 mRNA. By contrast, upregulating the expression of PD-L1 in ATG7-silenced cells mitigated the defects of autophagy induction, FOXO3A downregulation and miR-145 transcription attenuation. Overexpression of PD-L1 protein also enhanced bladder stem cell-like properties and the aggressiveness of bladder cancer cells (48).

PD-L1 is degraded on cancer cells via autophagy flux. Maher et al (49) found that PD-L1 protein expression was suppressed through induction of autophagy by RNAi-knockdown of Sigma 1 receptor and sigma non-opioid intracellular receptor 1 and small molecule inhibitor of Sigma 1 in triple-negative breast and androgen-independent prostate cancer cells that express Sigma 1. When administered alone, the Sigma inhibitor 1-(4-lodophenyl)-3-(2-adamantyl) guanidine (IPAG) induced autophagic flux, which increased LC3B and decreased cell surface PD-L1 expression in triple-negative breast and androgen-independent prostate cancer cells, hence suppressing the function of the PD-1-PD-L1 axis in T cells and cancer cells, indicating that autophagy can facilitate the degradation of PD-L1. In addition, when autophagy was 
inhibited by RNAi-knockdown of ATG5, ATG7 and the pharmacological inhibitors wortmannin and bafilomycin A1 (Baf A1), IPAG-mediated degradation of PD-L1 was blocked. This study performed confocal microscopy to observe the formation of autophagosomes and degradation of PD-L1 during IPAG treatment (43). Correspondingly, treatment with IPAG promoted the production of autophagosomes which was marked by green fluorescent protein (GFP)-LC3 and accelerated their co-localisation with PD-L1. Cotreatment with IPAG and Baf A1 contributed to enhanced amounts of GFP-LC3-positive puncta, which co-localised with PD-L1-containing autophagosomes. As aforementioned, in the case of using IPAG alone or in conjunction with Baf A1, the spots of PD-L1 were demonstrated to co-localise with autophagosomes (49). In summary, the aforementioned study illustrated that autophagy is an effective mechanism for IPAG to induce PD-L1 degradation in cells.

Liang et al (50) found that verteporfin, a small molecular inhibitor, inhibited the expression of PD-L1 both in vitro and in vivo via Golgi-related autophagy and blockade of the STAT1/interferon regulatory factor $1 /$ tripartite motif-containing 28 signalling pathway. Firstly, the study identified that verteporfin abolished both the mRNA and protein expression of PD-L1. Transmission electron microscopy revealed a sharp increase in autophagosomes in the cells following treatment with verteporfin, and increased LC3 lipidation and decreased p62/SQSTM1 expression (50). When autophagy was inhibited using chloroquine, the decreased expression of PD-L1 induced by verteporfin was reversed (50).

\section{Discussion}

$\mathrm{T}$ cell immunity is essential for homeostasis of the body, as it identifies antigens and kills cells with gene mutations or with aberrant pathology, which includes tumour cells. Unfortunately, excessive activation of uncontrolled $\mathrm{T}$ cells can also kill normal tissue cells, contributing to autoimmune diseases such as rheumatoid arthritis (51). Therefore, preventing autoimmunity by regulating activated $\mathrm{T}$ cells is an important feature of immune homeostasis. The co-inhibitory immune checkpoints, which include CTLA4-CD80, PD-1-PD-L1, galectin-9-T cell immunoglobulin mucin-3 and TCR-lymphocyte activation gene 3 , can regulate the activity of T cells under normal physiological conditions (52). However, upregulation of these inhibitory checkpoints leads to the immune microenvironment becoming immunosuppressed (53), which can cause immune tolerance and immune escape. The PD-1-PDL1 axis has been particularly identified as the most clinically significant, as antibodies against it have led to benefits in a variety of cancer types such as NSCLC, melanoma and gastric cancer $(9,11,17)$. Inhibition of PD-L1 expression on tumour cells heightens immunosurveillance and decreases the immune checkpoint function derived from PD-L1 (5). Hence, it is critical to explore the mechanisms that regulate the expression of PD-L1.

In the past ten years, the mechanisms that regulate PD-L1 expression via different pathways have been explored. First, the genomic alternation/rearrangements in chromosome 9p24.1, on which CD274 is located, have been identified to upregulate PD-L1 expression (54-58). It has been reported in the literature that amplification and mutations in the Janus kinase (JAK) family promote the upregulation of PD-L1 expression by inducing its mRNA expression $(55,59)$. The increase in activity of the JAK2/STAT signalling pathway resulting from gene mutations also increases PD-L1 expression $(55,59)$. DNA double-strand breaks also upregulate PD-L1 expression by activating the STAT signalling pathway via the kinases ATM/ATM and Rad3-related/checkpoint kinase $1(60,61)$. The expression of PD-L1 was induced by disrupting the CD274 3'UTR as well, by using CRISPR technology/Cas9 or miRNAs, such as miR-200, miR-34a, miR-152 and miR-424 (62-65). Epigenetic regulation, histone acetylation and methylation boost PD-L1 expression on melanoma and pancreatic cancer cells (66-68). In addition, oncogenic transcription factors, for example MYC, can combine with the PD-L1 promoter to enhance PD-L1 expression in hepatocellular carcinoma, human melanoma and NSCLC cell lines (69). Anaplastic lymphoma kinase can promote PD-L1 expression via STAT3 (70). Besides MYC and ALK, the mutated and amplified HIF $1 / 2 \alpha$ (hypoxia-inducible factor $1 / 2-\alpha$ ), NF- $\kappa B$, phosphatase and tensin homologue/PI3K, mitogen-activated protein kinase and epidermal growth factor receptor oncogenic pathways can upregulate PD-L1 mRNA expression on melanoma, ovarian cancers and lung squamous carcinoma cells (71-76). In addition, the IFN- $\gamma / \mathrm{JAK} / \mathrm{STAT} 1$ inflammatory pathway is used by cancer cells to enhance PD-L1 mRNA expression (77,78). Several inflammatory cytokines, such as Toll-like receptor 3, TNF- $\alpha$, transforming growth factor $\beta$, IFN- $\alpha / \beta$ and IL-4/6/17/27 have been demonstrated to upregulate PD-L1 mRNA expression on tumour cells or tumour-associated stromal cells (78-85). Post-transcriptional modifications, such as N-linked glycosylation (86), serine/threonine and tyrosine phosphorylation (87), polyubiquitination (88) and palmitoylation (89) have been found to serve significant roles in protein stability, as well as PD-L1 translocation regulation.

The present study demonstrated that autophagy can be induced by various molecules to downregulate PD-L1 through NF-kB, STAT3, HDAC6 or the degradation of autophagic flux. Whether autophagy can regulate PD-L1 expression through MYC, ALK, HIF1/2, several inflammatory cytokines, or other mechanisms remains to be investigated. Oncogenic pathways, such as PI3K/AKT and Ras/Raf/MEK/ERK, can induce autophagy and also regulate PD-L1 expression, which suggests that these pathways may regulate PD-L1 expression via autophagy.

mTOR is a major negative regulator of autophagy (90), which can be inhibited by RAP to reduce the expression of PD-L1 (91). When the PI3K/AKT signalling pathway, as the main modulator upstream of mTORC1 (92) is activated, this can phosphorylate tuberous sclerosis complex 2 to abolish the formation of the TSC1/2 complex, hence activating mTOR to inhibit autophagy (93). When the PI3K-AKT pathway is activated by epidermal growth factor and IFN- $\gamma$, it induces PD-L1 expression $(91,94,95)$. Furthermore, the PI3K-AKT pathway also can regulate PD-L1 expression in the absence of IFN- $\gamma$ in various cancer types, such as NSCLC, CRC, glioma, breast cancer and melanoma cells $(72,76,91,94,96)$, which suggests that this regulation occurs, at least in part, by changing the mRNA expression of PD-L1 $(74,97)$. 
In addition, the MEK-ERK signalling pathway has been identified frequently to be activated in a variety of cancer types such as hepatocellular carcinoma and colon cancer $(98,99)$. MEK-ERK is located on the outer surface of autophagosomes and promotes the production of Beclin1 protein by inducing the lipidation of LC3-I to LC3-II, hence enhancing autophagy (100). In addition, the MEK/ERK module promotes autophagy via the AMPK-MEK/ERK-TSC-mTOR signalling pathway (101). Continuous activation of the Ras/Raf/MEK/ERK pathway may enhance the autophagy flux in cells, and mRNA levels of LC3B and SQSTM1 are also increased (102). When the MEK-ERK pathway was inhibited by chemical or genetic inhibitors, PD-L1 transcription induced by IFN- $\gamma$ was inhibited in multiple myeloma cells (103). In concert with this, when the MEK-ERK signalling pathway was activated by phorbol myristate acetate, PD-L1 expression was enhanced. By contrast, when MEK was inhibited in tumours, PD-L1 expression was decreased in mouse-derived breast cancer cell lines $(103,104)$. Inhibition of the MEK-ERK signalling pathway abrogates increased PD-L1 expression stimulated by TLR ligands, which has been observed in various cancer cells and antigen presenting cells, such as myeloma, bladder cancer, lymphoma and dendritic cells $(94,97,98,103,105-107)$. Higher mutation rates of RAS and excessive activation of the RAS pathway have been demonstrated to increase PD-L1 expression among human lung and colorectal tumours (108). Besides, autophagy, as a pro-survival tumour mechanism, can be mediated by PD-L1 to escape the immune system. It has been reported that PD-L1 induces autophagy via mTORC signalling to promote the proliferation of ovarian cancer cells (109). As aforementioned, activated autophagy may reversely upregulate the PD-L1 expression through the ATG/autophagy/FOXO3A/miR-145 axis, forming a positive feedback loop to create a favourable environment for tumour progression.

As discussed above, the mechanisms that autophagy uses to regulate PD-L1 expression were reviewed. Autophagy can both positively and negatively regulate the PD-L1 expression on cancer cells. However, whether oncogenic pathways, such as PI3K/AKT and Ras/Raf/MEK/ERK, regulate PD-L1 expression via autophagy needs to be further elucidated. In addition, the mechanism by which autophagy effects PD-L1 expression remains to be further clarified in future studies.

In conclusion, autophagy can regulate PD-L1 expression in a number of cancer types via various mechanisms. Joint use of autophagy regulators and drugs targeting the PD-1/PD-L1 axis may enhance the therapeutic effect, hence improving the prognosis of patients with cancer.

\section{Acknowledgements}

Not applicable.

\section{Funding}

No funding was received.

\section{Availability of data and materials}

Not applicable.

\section{Authors' contributions}

LG was the main contributor to writing the manuscript and made substantial contributions to the conception and design. YC was responsible for the modification of the manuscript structure and language, was given final approval of the manuscript to be published and agreed to be accountable for all aspects of the work in ensuring that questions related to the accuracy or integrity of any part of the work are appropriately investigated and resolved. Both authors have read and approved the final manuscript. Data sharing is not applicable.

\section{Ethics approval and consent to participate}

Not applicable.

\section{Patient consent for publication}

Not applicable.

\section{Competing interests}

The authors declare that they have no competing interests.

\section{References}

1. Ishida Y, Agata Y, Shibahara K and Honjo T: Induced expression of PD-1, a novel member of the immunoglobulin gene superfamily, upon programmed cell death. EMBO J 11: 3887-3895, 1992.

2. Dong H, Zhu G, Tamada K and Chen L: B7-H1, a third member of the B7 family, co-stimulates T-cell proliferation and interleukin-10 secretion. Nat Med 5: 1365-1369, 1999.

3. Chen J, Jiang CC, Jin L and Zhang XD: Regulation of PD-L1: A novel role of pro-survival signalling in cancer. Ann Oncol 27: 409-416, 2016.

4. Boussiotis VA, Chatterjee P and Li L: Biochemical signaling of PD-1 on T cells and its functional implications. Cancer J 20: 265-271, 2014.

5. Wherry EJ: T cell exhaustion. Nat Immunol 12: 492-499, 2011.

6. Butte MJ, Keir ME, Phamduy TB, Sharpe AH and Freeman GJ: Programmed death-1 ligand 1 interacts specifically with the B7-1 costimulatory molecule to inhibit T cell responses. Immunity 27: 111-122, 2007.

7. Latchman YE, Liang SC, Wu Y, Chernova T, Sobel RA, Klemm M, Kuchroo VK, Freeman GJ and Sharpe AH: PD-L1-deficient mice show that PD-L1 on T cells, antigen-presenting cells, and host tissues negatively regulates T cells. Proc Natl Acad Sci USA 101: 10691-10696, 2004.

8. Brahmer JR, Rizvi NA, Lutzky J, Khleif S, Blake-Haskins A, Robbins XLB, Vasselli J, Ibrahim RA and Antonia SJ: Clinical activity and biomarkers of MEDI4736, an anti-PD-L1 antibody, in patients with NSCLC. J Clin Oncol 32 (15 Suppl): S8021, 2014.

9. Weber J, Mandala M, Del Vecchio M, Gogas HJ, Arance AM, Cowey CL, Dalle S, Schenker M, Chiarion-Sileni V, Marquez-Rodas I, et al: Adjuvant nivolumab versus ipilimumab in resected stage III or IV melanoma. N Engl J Med 377: 1824-1835, 2017.

10. Ansell SM, Lesokhin AM, Borrello I, Halwani A, Scott EC, Gutierrez M, Schuster SJ, Millenson MM, Cattry D, Freeman GJ, et al: PD-1 blockade with nivolumab in relapsed or refractory Hodgkin's lymphoma. N Engl J Med 372: 311-319, 2015.

11. Antonia SJ, Villegas A, Daniel D, Vicente D, Murakami S, Hui R, Yokoi T, Chiappori A, Lee KH, de Wit M, et al: Durvalumab after chemoradiotherapy in stage III non-small-cell lung cancer. N Engl J Med 377: 1919-1929, 2017.

12. Motzer RJ, Escudier B, McDermott DF, George S, Hammers HJ, Srinivas S, Tykodi SS, Sosman JA, Procopio G, Plimack ER, et al: Nivolumab versus everolimus in advanced renal-cell carcinoma. N Engl J Med 373: 1803-1813, 2015. 
13. Overman MJ, McDermott R, Leach JL, Lonardi S, Lenz HJ, Morse MA, Desai J, Hill A, Axelson M, Moss RA, et al: Nivolumab in patients with metastatic DNA mismatch repair-deficient or microsatellite instability-high colorecta cancer (CheckMate 142): An open-label, multicentre, phase 2 study. Lancet Oncol 18: 1182-1191, 2017.

14. Bellmunt J, de Wit R, Vaughn DJ, Fradet Y, Lee JL, Fong L, Vogelzang NJ, Climent MA, Petrylak DP, Choueiri TK, et al: Pembrolizumab as second-line therapy for advanced urothelial carcinoma. N Engl J Med 376: 1015-1026, 2017.

15. Kaufman HL, Russell J, Hamid O, Bhatia S, Terheyden P, D'Angelo SP, Shih KC, Lebbé C, Linette GP, Milella M, et al: Avelumab in patients with chemotherapy-refractory metastatic Merkel cell carcinoma: A multicentre, single-group, open-label, phase 2 trial. Lancet Oncol 17: 1374-1385, 2016.

16. El-Khoueiry AB, Sangro B, Yau T, Crocenzi TS, Kudo M, Hsu C, Kim TY, Choo SP, Trojan J, Welling TH Rd, et al: Nivolumab in patients with advanced hepatocellular carcinoma (CheckMate 040): An open-label, non-comparative, phase 1/2 dose escalation and expansion trial. Lancet 389: 2492-2502, 2017.

17. Fuchs CS, Doi T, Jang RW, Muro K, Satoh T, Machado M, Sun W, Jalal SI, Shah MA, Metges JP, et al: Safety and efficacy of pembrolizumab monotherapy in patients with previously treated advanced gastric and gastroesophageal junction cancer: Phase 2 clinical KEYNOTE-059 trial. JAMA Oncol 4: e180013, 2018.

18. Ferris RL, Blumenschein G Jr, Fayette J, Guigay J, Colevas AD, Licitra L, Harrington K, Kasper S, Vokes EE, Even C, et al: Nivolumab for recurrent squamous-cell carcinoma of the head and neck. N Engl J Med 375: 1856-1867, 2016.

19. Sunshine J and Taube JM: PD-1/PD-L1 inhibitors. Curr Opin Pharmacol 23: 32-38, 2015

20. Diem S, Hasan Ali O, Ackermann CJ, Bomze D, Koelzer VH, Jochum W, Speiser DE, Mertz KD and Flatz L: Tumor infiltrating lymphocytes in lymph node metastases of stage III melanoma correspond to response and survival in nine patients treated with ipilimumab at the time of stage IV disease. Cancer Immunol Immunother 67: 39-45, 2018

21. Kansy BA, Concha-Benavente F, Srivastava RM, Jie HB, Shayan G, Lei Y, Moskovitz J, Moy J, Li J, Brandau S, et al: PD-1 Status in $\mathrm{CD} 8^{+} \mathrm{T}$ cells associates with survival and anti-PD-1 therapeutic outcomes in head and neck cancer. Cancer Res 77 : 6353-6364, 2017

22. Hellmann MD, Callahan MK, Awad MM, Calvo E, Ascierto PA, Atmaca A, Rizvi NA, Hirsch FR, Selvaggi G, Szustakowski JD, et al: Tumor mutational burden and efficacy of nivolumab monotherapy and in combination with ipilimumab in small-cell lung cancer. Cancer Cell 33: 853-861.e4, 2018.

23. Meng X, Huang Z, Teng F, Xing L and Yu J: Predictive biomarkers in PD-1/PD-L1 checkpoint blockade immunotherapy. Cancer Treat Rev 41: 868-876, 2015.

24. Gandini S, Massi D and Mandalà M: PD-L1 expression in cancer patients receiving anti PD-1/PD-L1 antibodies: A systematic review and meta-analysis. Crit Rev Oncol Hematol 100: 88-98, 2016.

25. Wallis CJD, Lawson K, Butaney M, Satkunasivam R, Parikh J, Freedland SJ, Patel SP, Hamid O, Pal SK and Klaassen Z: Association between PD-L1 status and immune checkpoint inhibitor response in advanced malignancies: A systematic review and meta-analysis of overall survival data. Jpn J Clin Oncol 50: 800-809, 2020

26. Davis AA and Patel VG: The role of PD-L1 expression as a predictive biomarker: An analysis of all US Food and Drug Administration (FDA) approvals of immune checkpoint inhibitors. J Immunother Cancer 7: 278, 2019.

27. Wu P, Wu D, Li L, Chai Y and Huang J: PD-L1 and survival in solid tumors: A meta-analysis. PLoS One 10: e0131403, 2015.

28. Pyo JS, Kang G and Kim JY: Prognostic role of PD-L1 in malignant solid tumors: A meta-analysis. Int J Biol Markers 32: e68-e74, 2017.

29. Chen L and Han X: Anti-PD-1/PD-L1 therapy of human cancer: Past, present, and future. J Clin Invest 125: 3384-3391, 2015.

30. Topalian SL, Drake CG and Pardoll DM: Immune checkpoint blockade: A common denominator approach to cancer therapy. Cancer Cell 27: 450-461, 2015

31. Behrends C, Sowa ME, Gygi SP and Harper JW: Network organization of the human autophagy system. Nature 466: 68-76, 2010

32. Sosman JA, Kim KB, Schuchter L, Gonzalez R, Pavlick AC, Weber JS, McArthur GA, Hutson TE, Moschos SJ, Flaherty KT, et al: Survival in BRAF V600-mutant advanced melanoma treated with vemurafenib. N Engl J Med 366: 707-714, 2012 .
33. Kaushik S and Cuervo AM: The coming of age of chaperone-mediated autophagy. Nat Rev Mol Cell Biol 19: 365-381, 2018.

34. Dash S, Aydin Y and Moroz K: Chaperone-mediated autophagy in the liver: Good or bad? Cells 8: 1308, 2019.

35. Feng Y, He D, Yao Z and Klionsky DJ: The machinery of macroautophagy. Cell Res 24: 24-41, 2014.

36. Glick D, Barth S and Macleod KF: Autophagy: Cellular and molecular mechanisms. J Pathol 221: 3-12, 2010.

37. Shibutani ST and Yoshimori T: A current perspective of autophagosome biogenesis. Cell Res 24: 58-68, 2014.

38. Hailey DW, Rambold AS, Satpute-Krishnan P, Mitra K, Sougrat R, Kim PK and Lippincott-Schwartz J: Mitochondria supply membranes for autophagosome biogenesis during starvation. Cell 141: 656-667, 2010.

39. Huang J, Sun R, Qi X, Liu L, Yang Y and Sun B: Effect of autophagy on expression of neutrophil programmed death ligand-1 in mice with sepsis. Zhonghua Wei Zhong Bing Ji Jiu Yi Xue 31: 1091-1096, 2019 (In Chinese).

40. Booth L, Roberts JL, Poklepovic A and Dent P: (Pemetrexed + sildenafil), via autophagy-dependent HDAC downregulation, enhances the immunotherapy response of NSCLC cells. Cancer Biol Ther 18: 705-714, 2017.

41. Dent P, Booth L, Roberts JL, Poklepovic A and Hancock JF: (Curcumin + sildenafil) enhances the efficacy of 5FU and anti-PD1 therapies in vivo. J Cell Physiol 235: 6862-6874, 2020.

42. Chen MC, Lin YC, Liao YH, Liou JP and Chen CH: MPT0G612, a Novel HDAC6 inhibitor, induces apoptosis and suppresses IFN- $\gamma$-induced programmed death-ligand 1 in human colorectal carcinoma cells. Cancers (Basel) 11: 1617, 2019.

43. Booth L, Roberts JL, West C, Von Hoff D and Dent P: GZ17-6.02 initiates DNA damage causing autophagosome-dependent HDAC degradation resulting in enhanced anti-PD1 checkpoint inhibitory antibody efficacy. J Cell Physiol 235: 8098-8113, 2020.

44. Booth L, Roberts JL, Poklepovic A, Avogadri-Connors F, Cutler RE, Lalani AS and Dent P: HDAC inhibitors enhance neratinib activity and when combined enhance the actions of an anti-PD-1 immunomodulatory antibody in vivo. Oncotarget 8: 90262-90277, 2017.

45. Wang X, Wu WKK, Gao J, Li Z, Dong B, Lin X, Li Y, Li Y, Gong J, Qi C, et al: Autophagy inhibition enhances PD-L1 expression in gastric cancer. J Exp Clin Cancer Res 38: 140, 2019.

46. Buchser WJ, Laskow TC, Pavlik PJ, Lin HM and Lotze MT: Cell-mediated autophagy promotes cancer cell survival. Cancer Res 72: 2970-2979, 2012.

47. Tang D, Zhao D, Wu Y, Yao R, Zhou L, Lu L, Gao W and Sun Y: The miR-3127-5p/p-STAT3 axis up-regulates PD-L1 inducing chemoresistance in non-small-cell lung cancer. J Cell Mol Med 22: 3847-3856, 2018

48. Zhu J, Li Y, Luo Y, Xu J, Liufu H, Tian Z, Huang C, Li J and Huang C: A feedback loop formed by ATG7/autophagy, FOXO3a/miR-145 and PD-L1 regulates stem-like properties and invasion in human bladder cancer. Cancers (Basel) 11: 349, 2019.

49. Maher CM, Thomas JD, Haas DA, Longen CG, Oyer HM, Tong JY and Kim FJ: Small-molecule sigma1 modulator induces autophagic degradation of PD-L1. Mol Cancer Res 16: 243-255, 2018.

50. Liang J, Wang L, Wang C, Shen J, Su B, Marisetty AL, Fang D, Kassab C, Jeong KJ, Zhao W, et al: Verteporfin Inhibits PD-L1 through autophagy and the STAT1-IRF1-TRIM28 signaling axis, exerting antitumor efficacy. Cancer Immunol Res 8: 952-965, 2020.

51. Zhang N and Bevan MJ: CD8(+) T cells: Foot soldiers of the immune system. Immunity 35: 161-168, 2011.

52. Patel SP, Osada T, Osada K, Hurwitz H, Lyerly HK and Morse MA: Modulation of immune system inhibitory checkpoints in colorectal cancer. Curr Colorectal Cancer Rep 9: 391-397, 2013

53. Dunn GP, Old LJ and Schreiber RD: The three Es of cancer immunoediting. Annu Rev Immunol 22: 329-360, 2004

54. Cancer Genome Atlas Research Network: Comprehensive molecular characterization of gastric adenocarcinoma. Nature 513: 202-209, 2014

55. Green MR, Monti S, Rodig SJ, Juszczynski P, Currie T, O'Donnell E, Chapuy B, Takeyama K, Neuberg D, Golub TR, et al: Integrative analysis reveals selective 9p24.1 amplification, increased PD-1 ligand expression, and further induction via JAK2 in nodular sclerosing Hodgkin lymphoma and primary mediastinal large B-cell lymphoma. Blood 116 3268-3277, 2010. 
56. Ikeda S, Okamoto T, Okano S, Umemoto Y, Tagawa T, Morodomi Y, Kohno M, Shimamatsu S, Kitahara H, Suzuki Y, et al: PD-L1 is upregulated by simultaneous amplification of the PD-L1 and JAK2 genes in non-small cell lung cancer. J Thorac Oncol 11: 62-71, 2016.

57. Roemer MG, Advani RH, Ligon AH, Natkunam Y, Redd RA, Homer H, Connelly CF, Sun HH, Daadi SE, Freeman GJ, et al PD-L1 and PD-L2 genetic alterations define classical Hodgkin lymphoma and predict outcome. J Clin Oncol 34: 2690-2697, 2016.

58. Twa DD, Chan FC, Ben-Neriah S, Woolcock BW, Mottok A, Tan KL, Slack GW, Gunawardana J,Lim RS, McPherson AW, et al: Genomic rearrangements involving programmed death ligands are recurrent in primary mediastinal large B-cell lymphoma. Blood 123: 2062-2065, 2014.

59. Prestipino A, Emhardt AJ, Aumann K, O'Sullivan D, Gorantla SP, Duquesne S, Melchinger W, Braun L, Vuckovic S, Boerries M, et al: Oncogenic JAK2 ${ }^{\mathrm{V} 617 \mathrm{~F}}$ causes PD-L1 expression, mediating immune escape in myeloproliferative neoplasms Sci Transl Med 10: eaam7729, 2018

60. Sato H, Niimi A, Yasuhara T, Permata TBM, Hagiwara Y, Isono M, Nuryadi E, Sekine R, Oike T, Kakoti S, et al: DNA double-strand break repair pathway regulates PD-L1 expression in cancer cells. Nat Commun 8: 1751, 2017.

61. Sun LL, Yang RY, Li CW, Chen MK, Shao B, Hsu JM, Chan LC, Yang Y, Hsu JL, Lai YJ and Hung MC: Inhibition of ATR downregulates $\mathrm{PD}-\mathrm{L} 1$ and sensitizes tumor cells to T cell-mediated killing. Am J Cancer Res 8: 1307-1316, 2018.

62. Wang Q, Lin W, Tang X, Li S, Guo L, Lin Y and Kwok HF: The roles of microRNAs in regulating the expression of PD-1/PD-L1 immune checkpoint. Int J Mol Sci 18: 2540, 2017.

63. Xie G, Li W, Li R, Wu K, Zhao E, Zhang Y, Zhang P, Shi L, Wang D, Yin Y, et al: Helicobacter pylori promote B7-H1 expression by suppressing miR-152 and miR-200b in gastric cancer cells. PLoS One 12: e0168822, 2017.

64. Xu S, Tao Z, Hai B, Liang H, Shi Y, Wang T, Song W, Chen Y, OuYang J, Chen J, et al: MiR-424(322) reverses chemoresistance via T-cell immune response activation by blocking the PD-L1 immune checkpoint. Nat Commun 7: 11406, 2016.

65. Kataoka K, Shiraishi Y, Takeda Y, Sakata S, Matsumoto M, Nagano S, Maeda T, Nagata Y, Kitanaka A, Mizuno S, et al: Aberrant PD-L1 expression through 3'-UTR disruption in multiple cancers. Nature 534: 402-406, 2016

66. Deng S, Hu Q, Zhang H, Yang F, Peng C and Huang C: HDAC3 inhibition upregulates PD-L1 expression in B-cell lymphomas and augments the efficacy of anti-PD-L1 therapy. Mol Cancer Ther 18: 900-908, 2019.

67. Woods DM, Sodré AL, Villagra A, Sarnaik A, Sotomayor EM and Weber J: HDAC inhibition upregulates PD-1 ligands in melanoma and augments immunotherapy with PD-1 blockade. Cancer Immunol Res 3: 1375-1385, 2015.

68. Lu C, Paschall AV, Shi H, Savage N, Waller JL, Sabbatini ME, Oberlies NH, Pearce C and Liu K: The MLL1-H3K4me3 axis-mediated PD-L1 expression and pancreatic cancer immune evasion. J Natl Cancer Inst 109: djw283, 2017.

69. Casey SC, Tong L, Li Y, Do R, Walz S, Fitzgerald KN, Gouw AM, Baylot V, Gütgemann I, Eilers M and Felsher DW: MYC regulates the antitumor immune response through CD47 and PD-L1. Science 352: 227-231, 2016

70. Marzec M,Zhang Q, Goradia A, Raghunath PN, Liu X, Paessler M, Wang HY, Wysocka M, Cheng M, Ruggeri BA and Wasik MA: Oncogenic kinase NPM/ALK induces through STAT3 expression of immunosuppressive protein CD274 (PD-L1, B7-H1). Proc Natl Acad Sci USA 105: 20852-20857, 2008.

71. Akbay EA, Koyama S, Carretero J, Altabef A, Tchaicha JH, Christensen CL, Mikse OR, Cherniack AD, Beauchamp EM, Pugh TJ, et al: Activation of the PD-1 pathway contributes to immune escape in EGFR-driven lung tumors. Cancer Discov 3: $1355-1363,2013$

72. Atefi M, Avramis E, Lassen A, Wong DJ, Robert L, Foulad D, Cerniglia M, Titz B, Chodon T, Graeber TG, et al: Effects of MAPK and PI3K pathways on PD-L1 expression in melanoma. Clin Cancer Res 20: 3446-3457, 2014.

73. Barsoum IB, Smallwood CA, Siemens DR and Graham CH: A mechanism of hypoxia-mediated escape from adaptive immunity in cancer cells. Cancer Res 74: 665-674, 2014.

74. Jiang X, Zhou J, Giobbie-Hurder A, Wargo J and Hodi FS: The activation of MAPK in melanoma cells resistant to BRAF inhibition promotes PD-L1 expression that is reversible by MEK and PI3K inhibition. Clin Cancer Res 19: 598-609, 2013.
75. Peng J, Hamanishi J, Matsumura N, Abiko K, Murat K, Baba T, Yamaguchi K, Horikawa N, Hosoe Y, Murphy SK, et al: Chemotherapy induces programmed cell death-ligand 1 overexpression via the nuclear factor- $\kappa \mathrm{B}$ to foster an immunosuppressive tumor microenvironment in ovarian cancer. Cancer Res 75: 5034-5045, 2015.

76. Xu C, Fillmore CM, Koyama S, Wu H, Zhao Y, Chen Z, Herter-Sprie GS, Akbay EA, Tchaicha JH, Altabef A, et al: Loss of Lkb1 and pten leads to lung squamous cell carcinoma with elevated PD-L1 expression. Cancer Cell 25: 590-604, 2014.

77. Dong H, Strome SE, Salomao DR, Tamura H, Hirano F, Flies DB, Roche PC, Lu J, Zhu G, Tamada K, et al: Tumor-associated B7-H1 promotes T-cell apoptosis: A potential mechanism of immune evasion. Nat Med 8: 793-800, 2002.

78. Garcia-Diaz A, Shin DS, Moreno BH, Saco J, Escuin-Ordinas H, Rodriguez GA, Zaretsky JM, Sun L, Hugo W, Wang X, et al: Interferon receptor signaling pathways regulating PD-L1 and PD-L2 Expression. Cell Rep 19: 1189-1201, 2017.

79. Carbotti G, Barisione G, Airoldi I, Mezzanzanica D, Bagnoli M, Ferrero S, Petretto A, Fabbi M and Ferrini S: IL-27 induces the expression of IDO and PD-L1 in human cancer cells. Oncotarget 6: 43267-43280, 2015.

80. Lienlaf M, Perez-Villarroel P, Knox T, Pabon M, Sahakian E, Powers J, Woan K V, Lee C , Cheng F, Deng S, et al: Essential role of HDAC6 in the regulation of PD-L1 in melanoma. Mol Oncol 10: 735-750, 2016.

81. Ni XY, Sui HX, Liu Y, Ke SZ, Wang YN and Gao FG: TGF- $\beta$ of lung cancer microenvironment upregulates B7H1 and GITRL expression in dendritic cells and is associated with regulatory T cell generation. Oncol Rep 28: 615-621, 2012.

82. Pulko V, Liu X, Krco CJ, Harris KJ, Frigola X, Kwon ED and Dong H: TLR3-stimulated dendritic cells up-regulate B7-H1 expression and influence the magnitude of CD8 T cell responses to tumor vaccination. J Immunol 183: 3634-3641, 2009.

83. Quandt D, Jasinski-Bergner S, Müller U, Schulze B and Seliger B: Synergistic effects of IL-4 and TNFa on the induction of B7-H1 in renal cell carcinoma cells inhibiting allogeneic T cell proliferation. J Transl Med 12: 151, 2014.

84. Wang X, Yang L, Huang F, Zhang Q, Liu S, Ma L and You Z Inflammatory cytokines IL-17 and TNF- $\alpha$ up-regulate PD-L1 expression in human prostate and colon cancer cells. Immunol Lett 184: 7-14, 2017.

85. Zhang N, Zeng Y, Du W, Zhu J, Shen D, Liu Z and Huang JA: The EGFR pathway is involved in the regulation of PD-L1 expression via the IL-6/JAK/STAT3 signaling pathway in EGFR-mutated non-small cell lung cancer. Int J Oncol 49: 1360-1368, 2016.

86. Li CW, Lim SO, Xia W, Lee HH, Chan LC, Kuo CW, Khoo KH, Chang SS, Cha JH, Kim T, et al: Glycosylation and stabilization of programmed death ligand-1 suppresses T-cell activity. Nat Commun 7: 12632, 2016

87. Chan LC, Li CW, Xia W, Hsu JM, Lee HH, Cha JH, Wang HL, Yang WH, Yen EY, Chang WC, et al: IL-6/JAK1 pathway drives PD-L1 Y112 phosphorylation to promote cancer immune evasion. J Clin Invest 129: 3324-3338, 2019.

88. Mezzadra R, Sun C, Jae LT, Gomez-Eerland R, de Vries E, Wu W, Logtenberg MEW, Slagter M, Rozeman EA, Hofland I, et al: Identification of CMTM6 and CMTM4 as PD-L1 protein regulators. Nature 549: 106-110, 2017

89. Yang Y, Hsu JM, Sun L, Chan LC, Li CW, Hsu JL, Wei Y, Xia W, Hou J, Qiu Y and Hung MC: Palmitoylation stabilizes PD-L1 to promote breast tumor growth. Cell Res 29: 83-86, 2019.

90. Schmelzle T and Hall MN: TOR, a central controller of cell growth. Cell 103: 253-262, 2000.

91. Lastwika KJ, Wilson W III, Li QK, Norris J, Xu H, Ghazarian SR, Kitagawa H, Kawabata S, Taube JM, Yao S, et al: Control of PD-L1 expression by oncogenic activation of the AKT-mTOR pathway in non-small cell lung cancer. Cancer Res 76: 227-238, 2016.

92. Hay N: The Akt-mTOR tango and its relevance to cancer. Cancer Cell 8: 179-183, 2005.

93. Aoki M and Fujishita T: Oncogenic roles of the PI3K/AKT/mTOR Axis. Curr Top Microbiol Immunol 407: 153-189, 2017.

94. Song M, Chen D, Lu B, Wang C, Zhang J, Huang L, Wang X, Timmons CL, Hu J, Liu B, et al: PTEN Loss Increases PD-L1 protein expression and affects the correlation between PD-L1 expression and clinical parameters in colorectal cancer. PLoS One 8: e65821, 2013.

95. Zhang X, Zeng Y, Qu Q, Zhu J, Liu Z, Ning W, Zeng H, Zhang N, Du W, Chen C and Huang JA: PD-L1 induced by IFN- $\gamma$ from tumor-associated macrophages via the JAK/STAT3 and PI3K/AKT signaling pathways promoted progression of lung cancer. Int J Clin Oncol 22: 1026-1033, 2017. 
96. Parsa AT, Waldron JS, Panner A, Crane CA, Parney IF, Barry JJ, Cachola KE, Murray JC, Tihan T, Jensen MC, et al: Loss of tumor suppressor PTEN function increases B7-H1 expression and immunoresistance in glioma. Nat Med 13: 84-88, 2007.

97. Mittendorf EA, Philips AV, Meric-Bernstam F, Qiao N, Wu Y, Harrington S, Su X, Wang Y, Gonzalez-Angulo AM, Akcakanat A, et al: PD-L1 expression in triple-negative breast cancer. Cancer Immunol Res 2: 361-370, 2014.

98. Akula SM, Abrams SL, Steelman LS, Emma MR, Augello G, Cusimano A, Azzolina A, Montalto G, Cervello $M$ and McCubrey JA: RAS/RAF/MEK/ERK, $\mathrm{PI} 3 \mathrm{~K} / \mathrm{PTEN} / \mathrm{AKT} / \mathrm{mTORC} 1$ and TP53 pathways and regulatory miRs as therapeutic targets in hepatocellular carcinoma. Expert Opin Ther Targets 23: 915-929, 2019.

99. Wang Z, Ma L, Su M, Zhou Y, Mao K, Li C, Peng G, Zhou C, Shen B and Dou J: Baicalin induces cellular senescence in human colon cancer cells via upregulation of DEPP and the activation of Ras/Raf/MEK/ERK signaling. Cell Death Dis 9: 217, 2018.

100. Wang A, Zhang H, Liang Z, Xu K, Qiu W, Tian Y, Guo H, Jia J, Xing E, Chen R, et al: U0126 attenuates ischemia/reperfusion-induced apoptosis and autophagy in myocardium through MEK/ERK/EGR-1 pathway. Eur J Pharmacol 788: 280-285, 2016.

101. Wang J, Whiteman MW, Lian H, Wang G, Singh A, Huang D and Denmark T: A non-canonical MEK/ERK signaling pathway regulates autophagy via regulating Beclin 1 . J Biol Chem 284: 21412-21424, 2009.

102. Corcelle E, Nebout M, Bekri S, Gauthier N, Hofman P, Poujeol P, Fénichel P and Mograbi B: Disruption of autophagy at the maturation step by the carcinogen lindane is associated with the sustained mitogen-activated protein kinase/extracellular signal-regulated kinase activity. Cancer Res 66: 6861-6870, 2006.
103. Liu J, Hamrouni A, Wolowiec D, Coiteux V, Kuliczkowski K, Hetuin D, Saudemont A and Quesnel B: Plasma cells from multiple myeloma patients express B7-H1 (PD-L1) and increase expression after stimulation with IFN-\{gamma\} and TLR ligands via a MyD88-, TRAF6-, and MEK-dependent pathway. Blood 110: 296-304, 2007.

104. Loi S, Dushyanthen S, Beavis PA, Salgado R, Denkert C, Savas P, Combs S, Rimm DL, Giltnane JM, Estrada MV, et al: RAS/MAPK activation is associated with reduced tumor-infiltrating lymphocytes in triple-negative breast cancer: Therapeutic cooperation between MEK and PD-1/PD-L1 immune checkpoint inhibitors. Clin Cancer Res 22: 1499-1509, 2016.

105. Karakhanova S, Meisel S, Ring S, Mahnke K and Enk AH: ERK/p38 MAP-kinases and PI3K are involved in the differential regulation of B7-H1 expression in DC subsets. Eur J Immunol 40: 254-266, 2010.

106. Qian Y, Deng J, Geng L, Xie H, Jiang G, Zhou L, Wang Y, Yin S, Feng X, Liu J, et al: TLR4 signaling induces B7-H1 expression through MAPK pathways in bladder cancer cells. Cancer Invest 26: 816-821, 2008.

107. Yamamoto R, Nishikori M, Tashima M, Sakai T, Ichinohe T, Takaori-Kondo A, Ohmori K and Uchiyama T: B7-H1 expression is regulated by MEK/ERK signaling pathway in anaplastic large cell lymphoma and Hodgkin lymphoma. Cancer Sci 100: 2093-2100, 2009.

108. Coelho MA, de Carné Trécesson S, Rana S, Zecchin D, Moore C, Molina-Arcas M, East P, Spencer-Dene B, Nye E, Barnouin $\mathrm{K}$, et al: Oncogenic RAS signaling promotes tumor immunoresistance by stabilizing PD-L1 mRNA. Immunity 47: 1083-1099.e6, 2017.

109. Gao H, Zhang J and Ren X: PD-L1 regulates tumorigenesis and autophagy of ovarian cancer by activating mTORC signaling. Biosci Rep 39: BSR20191041, 2019. 